This document is the Accepted Manuscript version of a Published Work that appeared in final form in Analytical Chemistry, copyright (c) American Chemical Society after peer review and technical editing by the publisher. To access the final edited and published work see:

https://dx.doi.org/10.1021/acs. analchem.8b00389. 


\title{
Electrochromic Molecular Imprinting Sensor for visual and smartphone-based detections
}

\author{
Denise Capoferri ${ }^{a, b}+$, Ruslan Álvarez-Diduk ${ }^{a}$, Michele Del Carlo ${ }^{b}$, Dario Compagnone ${ }^{b}$, Arben \\ Merkoçi, ${ }^{a, c *}$ \\ ${ }^{a}$ Nanobioelectronics and Biosensor Group, Catalan Institute of Nanoscience and Nanotechnology (ICN2), CSIC. The \\ Barcelona Institute of Science and Technology, Campus UAB, Bellaterra, 08193, Barcelona, Spain. \\ ${ }^{b}$ Faculty of Biosciences and Technologies for Food, Agriculture and Environment, University of Teramo, via R. Balzarini 1, \\ 64100 Teramo, Italy \\ ${ }^{c}$ Catalan Institution for Research and Advanced Studies (ICREA), Pg. Lluís Companys 23, 08010 Barcelona, Spain
}

\begin{abstract}
Electrochromic effect and molecularly imprinted technology have been used to develop a sensitive and selective electrochromic sensor. The polymeric matrices obtained using the imprinting technology are robust molecular recognition elements and have the potential to mimic natural recognition entities with very high selectivity. The electrochromic behavior of iridium oxide nanoparticles (IrOx NPs) as physicochemical transducer together with a molecularly imprinted polymer (MIP) as recognition layer resulted in a fast and efficient translation of the detection event. The sensor was fabricated using screen-printing technology with indium tin oxide as a transparent working electrode; IrOx NPs where electrodeposited onto the electrode followed by thermal polymerization of polypyrrole in presence of the analyte (chlorpyrifos). Two different approaches were used to detect and quantify the pesticide; direct visual detection and smartphone imaging. Application of different oxidation potentials for 10 seconds resulted in color changes directly related to the concentration of the analyte. For smartphone imaging, at fixed potential, the concentration of the analyte was dependent on the color intensity of the electrode. The electrochromic sensor detects a highly toxic compound (chlorpyrifos) with a $100 \mathrm{fM}$ and $1 \mathrm{mM}$ dynamic range. So far, to the best of our knowledge, this is the first work where an electrochromic MIP sensor uses the electrochromic properties of IrOx to detect a certain analyte with high selectivity and sensitivity.
\end{abstract}

Development of sensors and biosensors has been very high in the last decades. Electrochemical sensors currently dominate the field but optical based detection techniques are considered more robust regarding bioaffinity. ${ }^{1}$ The combination of both (electro and optical properties) can improve the sensing capabilities in terms of sensitivity, selectivity, cost effectiveness and ease of use. ${ }^{2,3}$ In this regard, investigations on electrochromism phenomena appear very interesting; in fact, an electrochromic transducer takes advantage of the optical properties dependence of certain materials with the applied potential and current. ${ }^{4}$ Despite the latest efforts to develop electrochromic sensors, ${ }^{5-7}$ to the best of our knowledge, no device able to accomplish the aforementioned sensing ability has been reported. The combination of an electrochromic material with a biomimetic sensing layer seems promising to develop this kind of sensing assays.

Molecularly imprinted polymers (MIPs) are among the most used recognition elements in biomimetic sensors; in fact, recognition sites formed in the MIP can give excellent sensitivity and selectivity for the analyte. ${ }^{8-10}$ MIPs represent an alternative to natural receptors due to their robustness, versatility and cost effectiveness. ${ }^{11}$ Indeed, the chemical, thermal and mechanical stability, the facility of preparation and the relatively low cost of polymers compared to other biological recognition materials, as antibodies, make them attractive for several analytical applications. ${ }^{12}$ MIPs are synthesized by polymerization of selected functional monomers in the presence of a target analyte (template).
During the polymerization, a complex between the template and the functional monomer is formed resulting in a threedimensional polymer network, where the template molecules are incorporated into the polymer matrix. The template molecules are then extracted from the polymer matrix leaving the cavities inside, which are complementary in size, shape and functionality to the template. ${ }^{13,14}$ Hence, these cavities possess specificity and selectivity towards the identification of the template molecule only. ${ }^{15,16}$ Among the various types of conducting polymers, polypyrrole (PPy) is one of the best candidates to prepare molecular imprinting sensors because of its electrical conductivity, stability, excellent biocompatibility and facility for the immobilization of different compounds. ${ }^{12,17}$ ${ }^{21}$ In the last years, novel detection methods were developed by coupling the MIP with different nanocomposites and nanomaterials, such as palladium, platinum and silver nanoparticles, carbon nitride nanotubes, ${ }^{22-25}$ improving the analytical performances of the sensors. Particularly, for the analyte chosen in this research, a very low LOD was achieved in a voltammetric sensor using carbon nitride nanotubes decorated with graphene quantum dots. ${ }^{26}$

In this work, a novel MIP sensing device for chlorpyrifos detection has been developed, exploiting, for the first time, the electrochromism (EC) of iridium oxide (IrOx). Electrochromism is the phenomenon displayed by some materials of reversibly color change in response to an external applied potential. ${ }^{27-33}$ Several electrochromic materials have been reported, such as oxides of molybdenum, tungsten, 
titanium, iridium, and non-oxide materials as prussian blue tungsten sulfide, among others. ${ }^{27,34-36}$ Particularly, for IrOx our group has previously explored its electrochemical properties in sensing applications, ${ }^{37-41}$ but so far, its electrochromic properties have never been exploited with this purpose. IrOx is an electrochromic material which exhibits reversible and persistent changes in its optical properties ${ }^{42}$ generating large spectral shifts among its multiple oxidation states. Specifically, IrOx turns blue-black upon oxidation and becomes transparent upon reduction. ${ }^{43}$ For electrochromic applications, the working electrode should be transparent in order to assure visibility of the optical effect. Indium tin oxide (ITO) layers are mainly used since they represent a good compromise considering electrical conductivity, transparency and manufacturing costs. ${ }^{44}$

The combination of novel sensing technologies with smartphones facilitates the development of powerful lab-onsmartphone platforms. ${ }^{45,46}$ Nowadays, smartphones are widely integrated with sensors and used in biochemical detection; their portability and ubiquitous availability across the world allow a wide accessibility. ${ }^{45,47}$ Moreover, their use enable to get realtime and quantitative information which can be easily interpreted by the end-user. ${ }^{48}$ Smartphone-based sensing devices have great potential as point-of-care and point-of-need platforms for healthcare, food safety, environmental monitoring and biosecurity, especially in remote and rural areas. $^{49}$

The electrochromic sensor developed has an extremely low detection limit, can detect the analyte quickly with good selectivity in a wide dynamic range. In addition, the sensor was applied to spiked drinking water samples with satisfactory recoveries.

\section{EXPERIMENTAL SECTION}

\section{Chemicals and Apparatus}

Pyrrole, chlorpyrifos, chlorfenvinphos, dichlorvos, potassium hexachloroiridate (IV), Indium Tin Oxide (ITO) sheets, ethanol, sodium phosphate monobasic, sodium phosphate dibasic, acetone and hydrochloric acid were purchased from Sigma-Aldrich (Madrid, Spain). Potassium chloride and nitric acid $65 \%$ were purchased from PanReac AppliChem (Barcelona, Spain). Sodium hydroxide was obtained from Fluka Analytica (Munich, Germany). Stock solutions of pesticides were prepared in ethanol and stored at $20{ }^{\circ} \mathrm{C}$. All aqueous solutions were freshly prepared in Milli-Q water (Millipore). Pyrrole was distilled under vacuum until a colorless liquid was obtained, purged with nitrogen and kept in darkness at $-20{ }^{\circ} \mathrm{C}$. The ITO screen-printed electrodes (ITO SPEs) were home produced using a DEK 248 screen-printing machine. The SPEs were fabricated on adhesive plastic film (Aironfix) using carbon ink as the counter electrode (CE), $\mathrm{AgCl}$ ink as the reference electrode (RE) and the insulating ink as dielectric layer. High-resolution scanning electron microscopy (SEM) images were taken on a FEI Magellan 400L SEM (Hillsboro, OR). All SPEs pictures were taken with a Samsung Galaxy S7, in manual mode, manual focus, at ISO 100 and shutter speed 1/90 s, $12 \mathrm{Mp}$, ambient light conditions. Images were analyzed with the Image J App. Transmittance data were obtained using a Cary 4000 UV-Vis Spectrophotometer. Electrochemical measurements were carried out using an Autolab potentiostat-galvanostat PGSTAT $302 \mathrm{~N}$ controlled by a GPES 4.9.007 Software and were performed at room temperature using 0.1 M PBS (Phosphate
Buffer Solution) at $\mathrm{pH} 7$ containing $0.1 \mathrm{M} \mathrm{KCl}$ as a supporting electrolyte. ITO screen-printed electrodes were used for all measurements. The SPEs consist of a conventional three electrodes configuration with ITO modified with iridium oxide nanoparticles and MIP (3 mm diameter) as WE, carbon as CE and $\mathrm{AgCl}$ as $\mathrm{RE}$ (Figure 1A).

\section{Synthesis of iridium oxide nanoparticles (IrOx NPs)}

Fifty $\mathrm{mL}$ of $2 \mathrm{mM}$ aqueous $\mathrm{K}_{2} \mathrm{IrCl}_{6}$ solution was adjusted to pH 13 with 10 wt \% aqueous $\mathrm{NaOH}$ to obtain a yellow solution. It was then heated at $90{ }^{\circ} \mathrm{C}$ for 20 minutes. The resulting solution was kept in ice bath. The cold solution was adjusted to $\mathrm{pH} 1$ by rapidly adding $3 \mathrm{M} \mathrm{HNO}_{3}$ and was stirred continuously for 80 minutes until the solution became deep blue. The solution was adjusted to $\mathrm{pH} 7.5$ by addition of dilute 1.5 wt $\% \mathrm{NaOH}$ solution ${ }^{50}$ and stored in a refrigerator at $4{ }^{\circ} \mathrm{C}$.

\section{Fabrication of the ITO screen-printed electrodes (ITO SPEs)}

Small pieces of ITO $(4 \mathrm{~cm} \times 5 \mathrm{~cm})$ were pretreated by sonication for 10 minutes in each of the following solvents in an ultrasonic bath: soapy Milli-Q water, Milli-Q water, acetone and ethanol. Next, they were rinsed with Milli-Q water and dried with nitrogen. ${ }^{51}$ The ITO SPEs were home produced by a screen-printing technology.

\section{Deposition of the IrOx NPs on the ITO screen-printed electrodes}

Initially, the ITO SPEs were pretreated by cyclic voltammetry using a potential range between $-900 \mathrm{mV}$ and $+600 \mathrm{mV}$ in $\mathrm{PBS}(0.1 \mathrm{M})$ at $\mathrm{pH} 7$ containing $\mathrm{KCl}(0.1 \mathrm{M})$ at scan rate $100 \mathrm{mV} / \mathrm{s}$ until a reproducible voltammogram was observed. The electrodeposition of IrOx NPs was carried out by cyclic voltammetry in a $\mathrm{NaOH}$ solution $(55 \mathrm{mM})$. A drop of this solution containing the nanoparticles $(40 \mu \mathrm{L})$ was placed on the surface of the screen-printed electrode. After $10 \mathrm{~min}$, the electrodeposition was performed by voltage cycling (100 $\mathrm{mV} / \mathrm{s}$ ) between $-700 \mathrm{mV}$ and $+1000 \mathrm{mV}$ vs. $\mathrm{AgCl}$ for 50 cycles. ${ }^{52}$ The resulting deposited $\mathrm{IrOx}$ NPs were then rinsed with Milli-Q water and dried at room temperature before the production of MIP.

\section{Preparation of MIP and NIP/IrOx NPs - ITO screen- printed electrodes}

A drop $(5 \mu \mathrm{L})$ of the polymerization solution, pyrrole $0.5 \mathrm{M}$ (monomer) and chlorpyrifos $0.1 \mathrm{M}$ (template), was placed on the surface of the working electrode (IrOx NPs - ITO SPE) and was spin coated at $3000 \mathrm{rpm}$ for 60 seconds. Then the electrode was place in the oven at $90{ }^{\circ} \mathrm{C}$ for 1 minute to allow thermal polymerization to take place. The chlorpyrifos-polypyrrole MIP electrode, obtained after polymerization, was immersed in $\mathrm{HCl}$ solution $\mathrm{pH} 2$ with stirring for 15 minutes at room temperature to remove chlorpyrifos from the imprinted polymer (chlorpyrifos-free MIP electrode). The SPE was washed with Milli-Q water and allowed to dry at room temperature. Finally, for the rebinding step, the chlorpyrifosfree MIP electrode was dipped into chlorpyrifos solutions (2 $\mathrm{mL}$ ) at different concentrations (100 fM-1 mM) for 15 minutes (chlorpyrifos-rebinding MIP electrode) at room temperature. The SPE was washed with Milli-Q water and allowed to dry at room temperature before of the measurements. A control electrode (non-molecularly imprinted polymer electrode, NIP) 
was prepared under the same conditions but without the use of chlorpyrifos during the polymerization.

\section{Quantification of the analyte: visual and smartphone detections}

The color of the IrOx NPs was controlled applying a potential to the NPs using an Autolab/GPES interface. Electrochemical measurements were carried out placing a drop $(80 \mu \mathrm{L})$ of PBS at pH $7(0.1 \mathrm{M})$ containing $\mathrm{KCl}(0.1 \mathrm{M})$ on the surface of the MIP or NIP/IrOx NPs - ITO screen-printed electrodes and applying different potentials to oxidise (potential range between $+200 \mathrm{mV}$ and $+1000 \mathrm{mV}$ ) and to reduce (potential range between $-200 \mathrm{mV}$ and $-1000 \mathrm{mV}$ ) the IrOx NPs. For the visual detection, the time necessary to have the visual color change of the IrOx NPs (from transparent to blue-black) was used. For the smartphone detection, pictures of the screen printed-electrodes were taken with the smartphone, every second, during the IrOx NPs oxidation at an applied potential of $+300 \mathrm{mV}$ vs pseudo $\mathrm{Ag} / \mathrm{AgCl}$ reference electrode. Evaluation of the IrOx NPs color intensity was performed using Image $\mathrm{J}$.

\section{RESULTS AND DISCUSSION}

Chlorpyrifos (CPF) (IUPAC name: O,O-diethyl O-3,5,6trichloropyridin-2-yl phosphorothioate) was the target molecule selected in this study. It is a crystalline organophosphate pesticide (OP) extensively used in agriculture, households and urban insecticide applications. The sensor was developed using an ITO screen-printed electrode (SPE) modified with IrOx NPs and with a molecularly imprinted polypyrrole. Initially, $\mathrm{IrOx}$ NPs were electrodeposited using cyclic voltammetry and chlorpyrifospolypyrrole MIP films were then developed by thermal polymerization onto the surface of the working electrode (ITO), with pyrrole serving as the monomer and chlorpyrifos as the template (Figure 1A). The color change of the working electrode occurs both for positive and negative applied potentials. Figure 1A shows the operating principle of the sensor based on the decrease of conductivity of the MIP layer. Nanoparticles oxidation results to be hindered when a potential is applied in the presence of the analyte. Therefore, the electrochromic properties of the iridium oxide (color change) is limited. Two different approaches were set for the quantification of the pesticide: color intensity analysis using direct visual detection (Figure 1B) and image processing by a smartphone (Figure 1C).

Characterization of IrOx NPs and MIP/IrOx NPs - ITO screen-printed electrodes

The IrOx NPs were deposited onto the ITO SPEs by cyclic voltammetry $(100 \mathrm{mV} / \mathrm{s})$ between $-700 \mathrm{mV}$ and $+1000 \mathrm{mV}$ for 50 cycles. Figure S-1A shows an example of the growth of iridium oxide nanoparticles on the ITO SPE surface. Two cathodic and anodic peaks around $-400 \mathrm{mV}$ and $+500 \mathrm{mV}$ (versus $\mathrm{Ag} / \mathrm{AgCl}$ ) increase with the cycles as iridium oxide is electrodeposited on the electrode surface. Under these conditions, after 50 potential cycles, a uniform and adherent bluish film is observed on the electrode surface. In order to test the reversibility of the redox system, the IrOx NPs - ITO SPE was exposed to 25 oxidation scans (applied potential of +300 $\mathrm{mV}$ ) and 25 reduction scans (applied potential of $-300 \mathrm{mV}$ ). No significant difference in the current responses between the first and the last scan (Figure S-1B,C) was observed indicating the stability of the IrOx NPs film. Figure $\mathbf{2 A}$ shows the transmittance $(\%)$ spectra of ITO and IrOx NPs - ITO after the NPs oxidation and reduction states. As expected, the transmittance of reduced IrOx NPs (blue) was higher than oxidized NPs (red); the difference was up to $45 \%$ in the visible region. These results confirmed the color change between the oxidized and reduced states. Chronocoulometry measurements of IrOx NPs during the oxidation and reduction were also performed. Figures 2B and 2C report the charge $(\mathrm{mC})$ of the IrOx NPs for 100 seconds of both oxidation and reduction states. The NPs were also challenged with oxidation/reduction cycles using 10 seconds and 1 second switch between the two states. (Movie 1 and Movie 2 web enhanced objects) The results achieved demonstrated that the color changed rapidly, also for oxidation/reduction cycles with a switch of 1 second (Figure 2C).

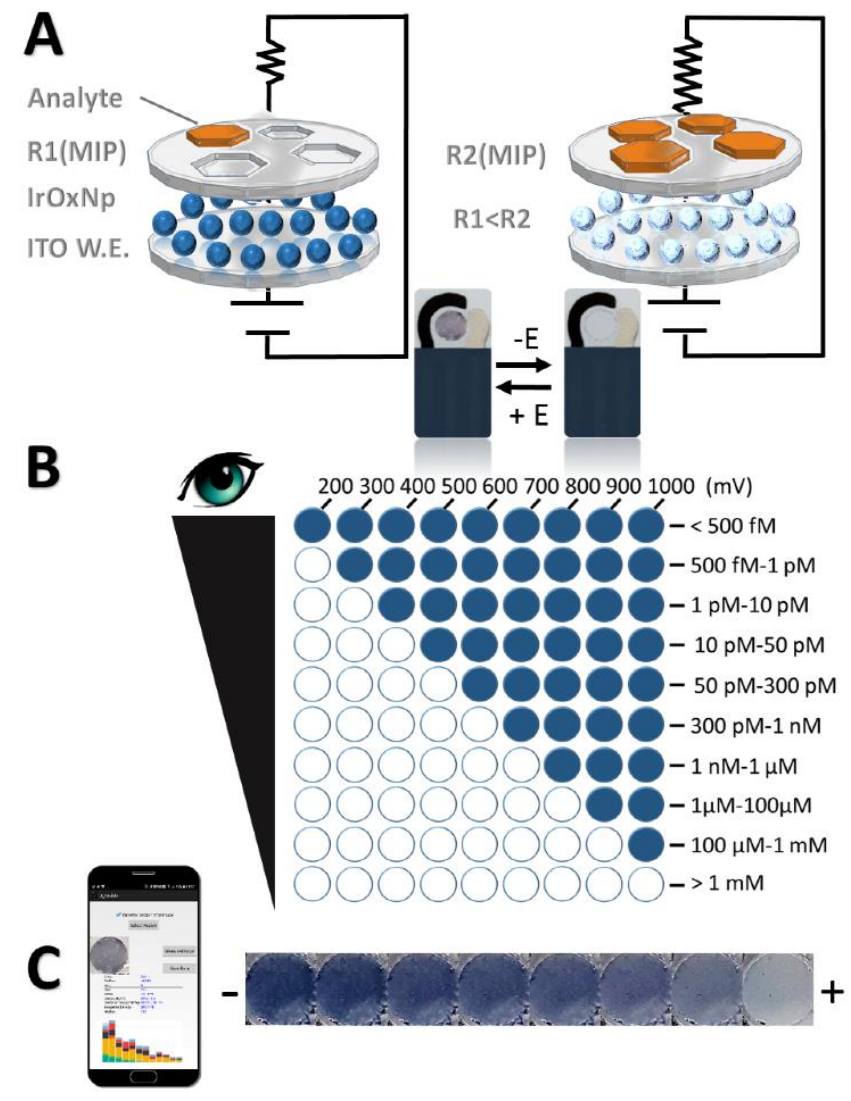

Figure 1. Scheme of the MIP/IrOx NPs - ITO SPEs structure, visual IrOx NPs color change (from blue-black to transparent); and working principle of the proposed sensor with different analyte amounts (A). Visual detection after 10 seconds of the application of different oxidation potentials and concentration ranges detected based on the number of colored electrodes (B); change of IrOx NPs color intensity at a fixed time and potential vs increasing amounts of the analyte (smartphone-based detection) (C).

The MIP sensor was prepared by the polymerization of pyrrole (monomer) in presence of chlorpyrifos (template molecule). Scanning electron microscopy was performed to characterize both the surface of IrOx NPs - ITO SPE and the MIP/IrOx NPs - ITO SPE. Figure S-1D reports the surface morphology of the IrOx NPs-modified electrode. IrOx NPs are observed confirming the electrochemical deposition of the nanoparticles on the electrode surface. MIP electrode surface appears rough (Figure S-1E) confirming the formation of the polymer. 

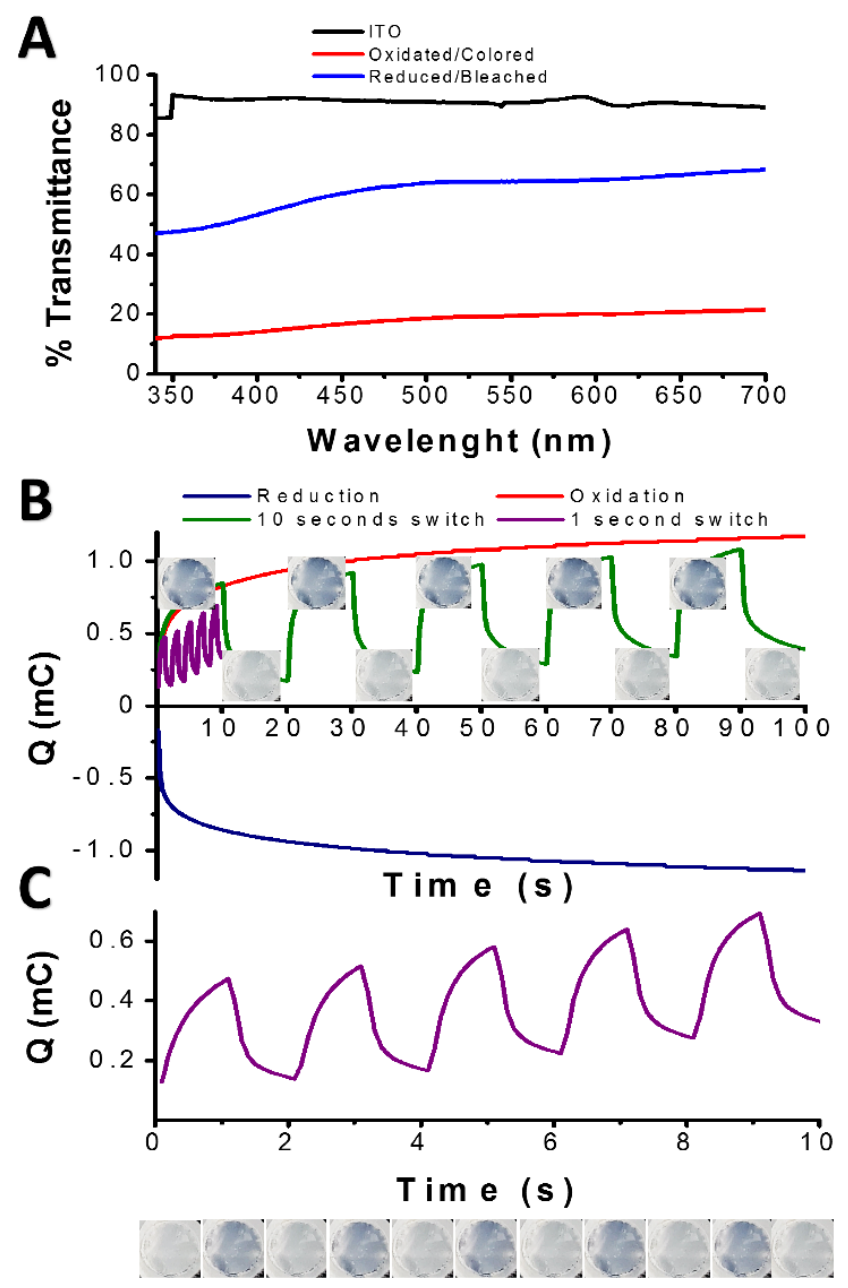

Figure 2. Transmittance (\%) spectra of ITO (black), oxidized IrOx NPs - ITO (red) and reduced IrOx NPs - ITO (blue) (A); charge (mC) of the IrOx NPs oxidation (red) and reduction (blue) during 100 seconds, oxidation/reduction cycles of IrOx NPs with a switch of 10 seconds (green) and 1 second (purple) (applied oxidation and reduction potentials of +500 $\mathrm{mV}$ and $-400 \mathrm{mV}$ ) (B); oxidation/reduction cycles of IrOx NPs with a switch of 1 second (applied oxidation and reduction potentials of $+500 \mathrm{mV}$ and $-400 \mathrm{mV}$ ) (C).

\section{Detection of chlorpyrifos at the MIP/IrOx NPs - ITO screen-printed electrodes}

The MIP sensor was tested with different amounts of chlorpyrifos (100 $\mathrm{fM}-1 \mathrm{mM})$; the electrochromic properties are correlated with the concentration of the pesticide. Figure 3A reports the time dependence vs potential of the chlorpyrifos concentration (potential range between +200 and $+1000 \mathrm{mV}$ ). The height of the bars represents the time required to appreciate a visual color change of the IrOx NPs. For the sake of clarity, a potential of $+300 \mathrm{mV}$ (yellow bars) was selected to report the actual data. It is clear from the figure that the amount of the analyte can be determined measuring the time. As the concentration increases, the time to observe a color change in the IrOx NPs is delayed. The increased amount of analyte inside the polymer cavities reduce the conductivity of the polypyrrole. This was confirmed by the behavior observed at fixed concentration of the analyte. The higher the potential, in this case, the lower the time delay needed to observe a color change. All values were taken from Table S-1.

Looking at the data, similar considerations can be done for another method of quantification. Figure 3A (orange bars) and Figure 1B show how the concentration of the analyte can be also obtained at fixed time. After 10 seconds from the application of the oxidation potential (potential in the +200 /+1000 $\mathrm{mV}$ range), it was possible to distinguish different pesticide concentrations ( $500 \mathrm{fM}-1 \mathrm{mM}$ range). This allows the use for the detection of the analyte of a series of electrodes each at a different applied potential. For instance, in the case that all electrodes change the color after 10 seconds of the application of the different potentials, the expected concentration has to be $<500 \mathrm{fM}$. However, if a color change is observed in all electrodes except the first, the concentration range of pesticide has to be between $500 \mathrm{fM}-1 \mathrm{pM}$. The same applies for the rest of the electrodes (Figure 1B) until the last row, where no color change means concentration of analyte above $1 \mathrm{mM}$.

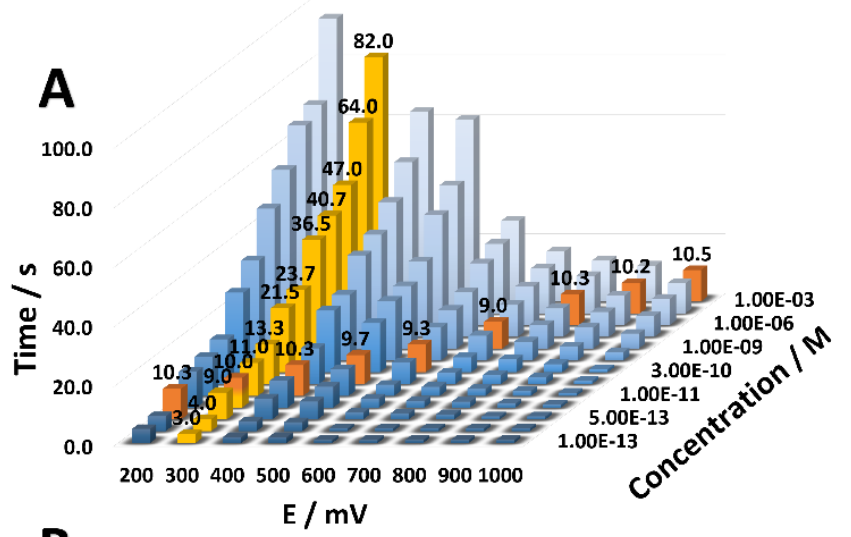

B

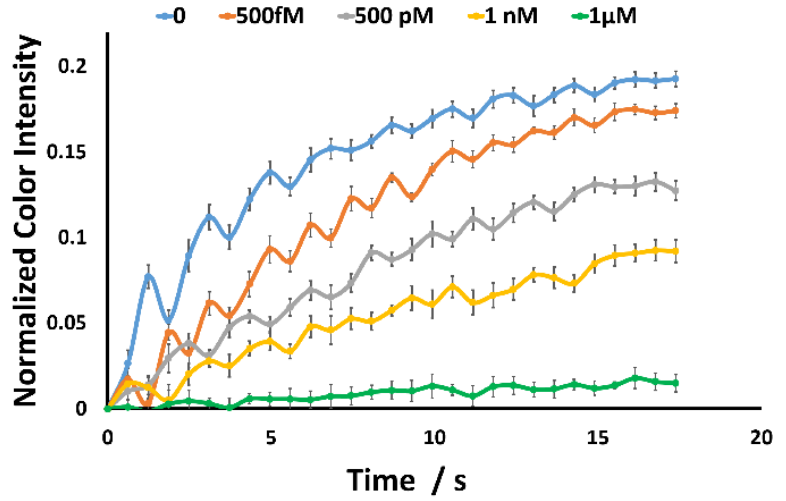

Figure 3. Time needed to reach visual color change of the IrOx NPs. Setting the oxidation potential, different chlorpyrifos concentrations from $100 \mathrm{fM}$ to $1 \mathrm{mM}$ were tested (A); normalized IrOx NPs color intensity (Image J app) at different concentrations of chlorpyrifos vs time (applied oxidation potential of $+300 \mathrm{mV}$ ) (B).

Pictures of the working electrodes were taken to analyze the color intensity change as a function of the analyte concentration. Images were taken with a smartphone during the oxidation of the IrOx NPs (at applied potential) and analyzed using Image $\mathbf{J}$ software. Using this approach, with the increase of the oxidation time, for every concentration tested $(500 \mathrm{fM}$, $500 \mathrm{pM}, 1 \mathrm{nM}$ and $1 \mu \mathrm{M})$, the working electrode color intensity increased. The IrOx NPs normalized color intensity is reported in Figure 3B. Moreover, the color intensity change 
stopped after about 16 seconds and remained stable. There was a difference in the intensity for the different concentrations, in particular the values decreased with higher amounts of pesticide (Figures $\mathbf{1 C}$ and $\mathbf{3 B}$ ). These results indicate that the decrease of the IrOx NPs normalized color intensity can be used to detect the analyte quantitatively.

According to these results, it is possible to assume that there is an inverse correlation between the generated current response and pesticide concentration. The higher the analyte concentration used the lower the oxidation current during the color change of the IrOx NPs. This can be also explained by reduction of the conductivity of polypyrrole due to rebinding of the analyte in the polymer free cavities (Figure S-2). The dose-response logarithmic curves obtained for time and current are reported in Figure S-3. The responses increase in a wide range between $100 \mathrm{fM}$ and $1 \mathrm{mM}$. The sensor was able to detect chlorpyrifos at low concentrations with a detection limit (LOD $=0.1 \mathrm{pM})$ lower than those obtained with other MIP sensors. ${ }^{26,53-56}$ In the case of the NIP sensor, the responses were very small and were independent of the amount of analyte used (Figure S-4). This can be attributed to the non-specific adsorption of chlorpyrifos onto the polypyrrole. For repeatability and reproducibility tests, once the color change was observed, the current and time responses were respectively measured. Repeatability, calculated for $300 \mathrm{fM}$ and for $1 \mu \mathrm{M}$ of analyte using the same electrode $(n=3)$ gave, respectively, RSDs of $0.6 \%$ and $0.7 \%$ (current response) and RSDs of $0.2 \%$ and $3.3 \%$ (time response). Measurements using the same analyte concentrations with three different sensors gave, respectively, RSDs of $13.4 \%$ and $3.4 \%$ (current response) and RSDs of $18.8 \%$ and $7.3 \%$ (time response).

\section{Selectivity study}

The selectivity of the developed sensor for chlorpyrifos was tested challenging the electrode with two other organophosphates (chlorfenvinphos and dichlorvos) in the 500 $\mathrm{fM}$ to $1 \mathrm{mM}$ range by direct visual detection. $\Delta \mathrm{I}(\%)$ for the interferents was lower than for chlorpyrifos. At concentrations of $500 \mathrm{fM}, 500 \mathrm{pM}$ and $1 \mathrm{nM}$, the response of the interferents was between $13 \%$ and $24 \%$ with respect to chlorpyrifos. The responses were higher for concentrations of $1 \mu \mathrm{M}$ and $1 \mathrm{mM}$, being for chlorfenvinphos $34 \%$ and $44 \%$ respectively, and for dichlorvos $38 \%$ and $44 \%$, compared with the target analyte (Figure 4A). $\Delta \mathrm{t}$ (s) for the interferents was $20 \%$ at $500 \mathrm{fM}$ and was between $12 \%$ and $17 \%$ at $500 \mathrm{pM}$ and $1 \mathrm{nM}$, compared with chlorpyrifos. At higher concentrations ( $1 \mu \mathrm{M}$ and $1 \mathrm{mM})$, $\Delta \mathrm{t}$ (s) responses were $15 \%$ and $21 \%$ (chlorfenvinphos) and $17 \%$ and $22 \%$ (dichlorvos) with respect to the target analyte (Figure 4B). The results obtained indicate that the electrochromic sensor exhibits a good selectivity to chlorpyrifos compared to other organophosphates.

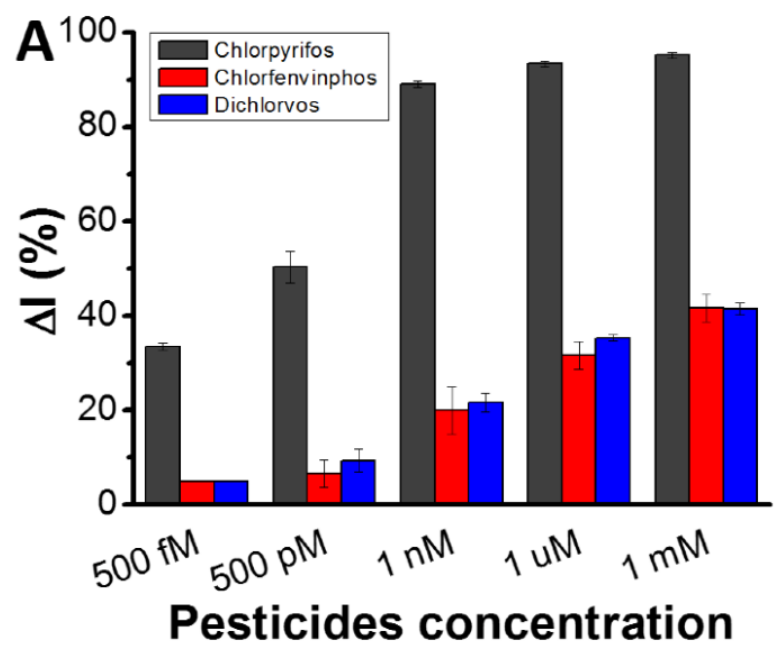

B

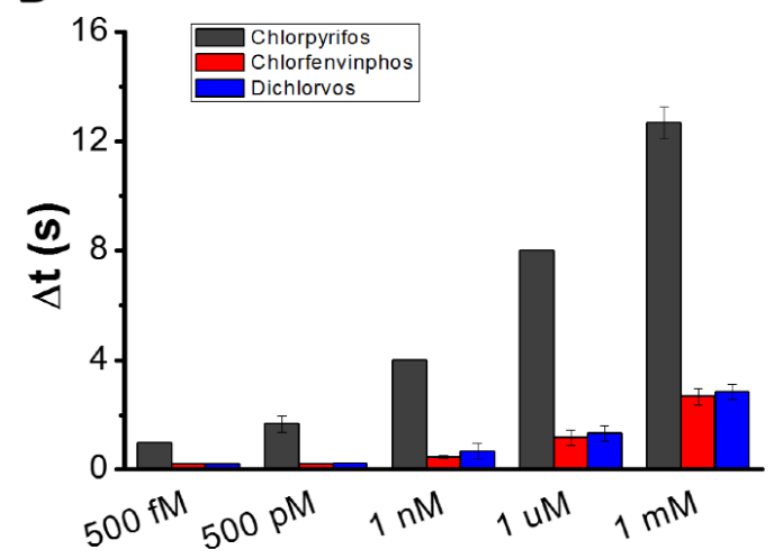

Pesticides concentration

Figure 4. $\Delta \mathrm{I}(\%)$ and $\Delta \mathrm{t}(\mathrm{s})$ of the MIP electrode for the chlorpyrifos, chlorfenvinphos and dichlorvos (A, B). $\Delta \mathrm{I}$ represents the difference between the oxidation currents (measured during the visual color change of the IrOx NPs, applied potential of $+500 \mathrm{mV}$ ) after the washing step and after the rebinding step in different concentrations of chlorpyrifos, chlorfenvinphos and dichlorvos solutions. $\Delta \mathrm{t}$ represents the difference in the time, to reach the visual color change of the IrOx NPs (applied potential of $+1000 \mathrm{mV}$ ), between the rebinding step in different concentrations of chlorpyrifos, chlorfenvinphos and dichlorvos solutions and the washing step.

\section{Analysis of chlorpyrifos in spiked drinking water samples}

In order to demonstrate the practical utility of the sensor, drinking water samples were spiked with different concentrations of chlorpyrifos to have final concentrations of $500 \mathrm{fM}, 500 \mathrm{pM}, 1 \mathrm{nM}, 1 \mu \mathrm{M}$ and $1 \mathrm{mM}$. The MIP/IrOx NPs ITO SPEs were immersed into the spiked solutions $(2 \mathrm{~mL})$ for 15 minutes at room temperature. After the incubation, the electrodes were washed with Milli-Q water and allowed to dry at room temperature before measuring current and time. As reported in Tables $\mathbf{S - 2}$ and $\mathbf{S - 3}$, the recovery values obtained were in the range of $94 \%-107 \%$ using the current response and in the range $81 \%-103 \%$ using the time response, which demonstrates the viability of the developed sensor to detect chlorpyrifos in real samples. 


\section{CONCLUSIONS}

In this work, the electrochromic properties of $\mathrm{IrOx}$ nanoparticles were exploited for the first time to develop a selective and sensitive sensor combining a transparent ITO electrode and a MIP. The dependence of time and oxidation potential to achieve the IrOx NPs color change as a function of the concentration provides a great versatility to the sensor. Sensor ability to detect the analyte was successfully proven by two approaches (visual and smartphone-based detection). The time response could be used to develop a device for fast and in situ screening analysis using a set of electrodes each with a different applied potential. In this way, by setting the time at 10 seconds and increasing the oxidation potential it is possible to assess the analyte concentration range. The color intensity response can be used for the analyte detection using a smartphone and measuring the IrOx NPs color intensity. The developed sensor was able to detect chlorpyrifos at very low concentrations, the detection limit is lower (LOD $=0.1 \mathrm{pM}$ ) than those obtained in previously reported MIP sensors. Besides, it exhibits an excellent repeatability, good reproducibility and selectivity. In summary, a fast, low cost, portable, disposable and easy to use sensor was developed taking advantage of the electrochromic properties of IrOx. The novel electrochromic sensor offers new perspectives for the sensitive and selective detection of different compounds particularly in cases where expensive and sophisticated instrumentation is not available. It can be very useful for cheap, rapid, real time and in situ screening analysis either in food and/or environmental samples with versatile applications.

\section{ASSOCIATED CONTENT}

\section{Web enhanced objects}

Movie 1: clip of oxidation/reduction cycles for 10 seconds

Movie 2: clip of oxidation/reduction cycles for 1 second

\section{Supporting Information}

Supporting Information Available:

Figure S-1: consecutive cyclic voltammograms of the $\mathrm{IrOx}$ NPs deposition on the ITO SPE (A); IrOx NPs stress test (B, C); SEM images of IrOx NPs - ITO SPE and MIP/IrOx NPs - ITO SPE (D, E).

Table S-1: time necessary to reach the visual color change of IrOx NPs for different oxidation potentials applied after the rebinding in different chlorpyrifos concentrations.

Figure S-2: current during the $\mathrm{IrOx}$ NPs color change vs pesticide concentrations.

Figure S-3: dose - response $(\Delta \mathrm{I} \% ; \Delta \mathrm{t}(\mathrm{s}))$ logarithmic curves.

Figure S-4: $\Delta \mathrm{I}(\%)$ and $\Delta \mathrm{t}(\mathrm{s})$ of the MIP and NIP electrodes.

Tables S-2 and S-3: recovery values of chlorpyrifos in spiked drinking water samples $(n=3)$ using the current and time responses.

This material is available free of charge on the ACS Publications website at DOI....

\section{AUTHOR INFORMATION}

Corresponding Author

*arben.merkoci@icn2.cat

\section{Author Contributions}

These authors contributed equally to this work. All authors have reviewed and approved the final version of the manuscript.

\section{Notes}

The authors declare no competing financial interest.

\section{ACKNOWLEDGMENT}

This work was supported by the Spanish MINECO under project MAT2014-52485-P. This work is also funded by the CERCA Programme / Generalitat de Catalunya. ICN2 is supported by the Severo Ochoa program from Spanish MINECO (Grant No. SEV-2013-0295). R. Alvarez acknowledges the financial support from CONACYT (Mexico).

\section{REFERENCES}

(1) Turner, A. P. Chem. Soc. Rev. 2013, 42, 3184-3196.

(2) Juan-Colás, J.; Johnson, S.; Krauss, T. F. Sensors 2017, 17, 2047.

(3) Colás, J. J. In Dual-Mode Electro-photonic Silicon Biosensors; Springer, 2017, pp 59-93.

(4) DeLongchamp, D. M.; Hammond, P. T. Chem. Mater. 2004, 16, 4799-4805.

(5) Pellitero, M. A.; Guimerà, A.; Kitsara, M.; Villa, R.; Rubio, C.; Lakard, B.; Doche, M.-L.; Hihn, J.-Y.; del Campo, F. J. Chem. Sci. 2017, 8, 1995-2002.

(6) Gao, C.; Wang, Y.; Yuan, S.; Xue, J.; Cao, B.; Yu, J. Biosens. Bioelectron. 2017, 90, 336-342.

(7) Xu, W.; Fu, K.; Bohn, P. W. ACS Sens. 2017, 2, 1020-1026.

(8) Ding, X.; Heiden, P. A. Macromol. Mater. Eng. 2014, 299, 268-282.

(9) Merkoci, A.; Alegret, S. TrAC, Trends Anal. Chem. 2002, 21, 717-725.

(10) Zamora-Gálvez, A.; Mayorga-Matinez, C. C.; Parolo, C.; Pons, J.; Merkoçi, A. Electrochem. Commun. 2017, 82, 6-11.

(11) Mazzotta, E.; Turco, A.; Chianella, I.; Guerreiro, A.; Piletsky, S. A.; Malitesta, C. Sens. Actuators, B 2016, 229, 174-180.

(12) da Silva, H.; Pacheco, J. G.; Magalhães, J. M.; Viswanathan, S.; Delerue-Matos, C. Biosens. Bioelectron. 2014, 52, 56-61.

(13) Chen, N.; Chen, L.; Cheng, Y.; Zhao, K.; Wu, X.; Xian, Y. Talanta 2015, 132, 155-161.

(14) Rotariu, L.; Lagarde, F.; Jaffrezic-Renault, N.; Bala, C. TrAC, Trends Anal. Chem. 2016, 79, 80-87.

(15) Roy, E.; Patra, S.; Tiwari, A.; Madhuri, R.; Sharma, P. K. Biosens. Bioelectron. 2017, 89, 234-248.

(16) Li, Y.; Liu, Y.; Liu, J.; Liu, J.; Tang, H.; Cao, C.; Zhao, D.; Ding, Y. Sci. Rep. 2015, 5, 7699.

(17) Jara-Ulloa, P.; Salgado-Figueroa, P.; Moscoso, R.; Squella, J. A. J. Electrochem. Soc. 2013, 160, H243-H246.

(18) Lian, W.; Liu, S.; Wang, L.; Liu, H. Biosens. Bioelectron. 2015, 73, 214-220.

(19) Gupta, V. K.; Yola, M. L.; Özaltın, N.; Atar, N.; Üstündağ, Z.; Uzun, L. Electrochim. Acta 2013, 112, 37-43.

(20) Capoferri, D.; Del Carlo, M.; Ntshongontshi, N.; Iwuoha, E. I.; Compagnone, D. In Convegno Nazionale Sensori; Springer, 2016, pp 157-162.

(21) Capoferri, D.; Del Carlo, M.; Ntshongontshi, N.; Iwuoha, E.; Sergi, M.; Di Ottavio, F.; Compagnone, D. Talanta 2017, 174, 599-604.

(22) Beytur, M.; Kardaş, F.; Akyıldırım, O.; Özkan, A.; Bankoğlu, B.; Yüksek, H.; Yola, M. L.; Atar, N. J. Mol. Liq. 2018, 251, 212217.

(23) Yola, M. L. t.; Atar, N. Ind. Eng. Chem. Res. 2017, 56, 76317639. 
(24) Akyıldırım, O.; Kardaş, F.; Beytur, M.; Yüksek, H.; Atar, N.; Yola, M. L. J. Mol. Liq. 2017, 243, 677-681.

(25) Yola, M. L.; Atar, N. J. Electrochem. Soc. 2018, 165, H1-H9. (26) Yola, M. L.; Atar, N. J. Electrochem. Soc. 2017, 164, B223B229.

(27) Granqvist, C. G. Handbook of inorganic electrochromic materials; Elsevier, 1995.

(28) Jiang, F.; Zheng, T.; Yang, Y. J. Non-Cryst. Solids 2008, 354, 1290-1293

(29) Park, S.-I.; Quan, Y.-J.; Kim, S.-H.; Kim, H.; Kim, S.; Chun, D.-M.; Lee, C. S.; Taya, M.; Chu, W.-S.; Ahn, S.-H. Int. J. Pr. Eng. Man.-GT 2016, 3, 397-421.

(30) Nam, Y. S.; Park, H.; Magyar, A. P.; Yun, D. S.; Pollom, T. S.; Belcher, A. M. Nanoscale 2012, 4, 3405-3409.

(31) Niwa, T.; Takai, O. Jpn. J. Appl. Phys. 2010, 49, 105802.

(32) Nishio, K.; Watanabe, Y.; Tsuchiya, T. Thin Solid Films 1999, 350, 96-100.

(33) Patil, P.; Kawar, R.; Sadale, S. Electrochim. Acta 2005, 50, 2527-2532.

(34) Monk, P. M.; Ali, T.; Partridge, R. D. Solid State Ionics 1995, $80,75-85$.

(35) Maruyama, T.; Kanagawa, T. J. Electrochem. Soc. 1996, 143 , $1675-1677$

(36) Kanoh, H.; Hirotsu, T.; Ooi, K. J. Electrochem. Soc. 1996, 143, 905-907.

(37) Rivas, L.; Mayorga-Martinez, C. C.; Quesada-González, D.; Zamora-Gálvez, A.; de la Escosura-Muñiz, A.; Merkoçi, A. Anal. Chem. 2015, 87, 5167-5172.

(38) Rivas, L.; de la Escosura-Muñiz, A.; Pons, J.; Merkoçi, A. Electroanalysis 2014, 26, 1287-1294.

(39) Mayorga-Martinez, C. C.; Pino, F.; Kurbanoglu, S.; Rivas, L.; Ozkan, S. A.; Merkoçi, A. J. Mater. Chem. B 2014, 2, 2233-2239.

(40) Mayorga-Martinez, C. C.; Chamorro-García, A.; Serrano, L.; Rivas, L.; Quesada-Gonzalez, D.; Altet, L.; Francino, O.; Sánchez, A.; Merkoçi, A. J. Mater. Chem. B 2015, 3, 5166-5171.
(41) Kurbanoglu, S.; Rivas, L.; Ozkan, S. A.; Merkoçi, A. Biosens. Bioelectron. 2017, 88, 122-129.

(42) Ito, S.; Abe, Y.; Kawamura, M.; Kim, K. H. J. Vac. Sci. Technol., B: Nanotechnol. Microelectron.: Mater., Process., Meas., Phenom. 2015, 33, 041204.

(43) Abe, Y.; Ito, S.; Kim, K. H.; Kawamura, M.; Kiba, T. J. Mater. Sci. Res. 2016, 6, 44.

(44) Bange, K.; Gambke, T. Adv. Mater. 1990, 2, 10-16.

(45) Rateni, G.; Dario, P.; Cavallo, F. Sensors 2017, 17, 1453.

(46) Quesada-González, D.; Merkoçi, A. Biosens. Bioelectron. 2017, 92, 549-562.

(47) Zhang, D.; Liu, Q. Biosens. Bioelectron. 2016, 75, 273-284.

(48) Álvarez-Diduk, R.; Orozco, J.; Merkoçi, A. Sci. Rep. 2017, 7.

(49) Roda, A.; Michelini, E.; Zangheri, M.; Di Fusco, M.; Calabria, D.; Simoni, P. TrAC, Trends Anal. Chem. 2016, 79, $317-$ 325.

(50) Zhao, Y.; Hernandez-Pagan, E. A.; Vargas-Barbosa, N. M.; Dysart, J. L.; Mallouk, T. E. J. Phys. Chem. Lett. 2011, 2, 402406.

(51) Ballarin, B.; Cassani, M. C.; Scavetta, E.; Tonelli, D Electrochim. Acta 2008, 53, 8034-8044.

(52) Casella, I. G.; Contursi, M.; Toniolo, R. J. Electroanal. Chem. 2015, 736, 147-152.

(53) Uygun, Z. O.; Dilgin, Y. Sens. Actuators, B 2013, 188, 78-84. (54) Wang, P.; Dai, W.; Ge, L.; Yan, M.; Ge, S.; Yu, J. Analyst 2013, 138, 939-945.

(55) Yao, G.-H.; Liang, R.-P.; Huang, C.-F.; Wang, Y.; Qiu, J.-D. Anal. Chem. 2013, 85, 11944-11951.

(56) Ren, X.; Liu, H.; Chen, L. Microchim. Acta 2015, 182, $193-$ 200. 


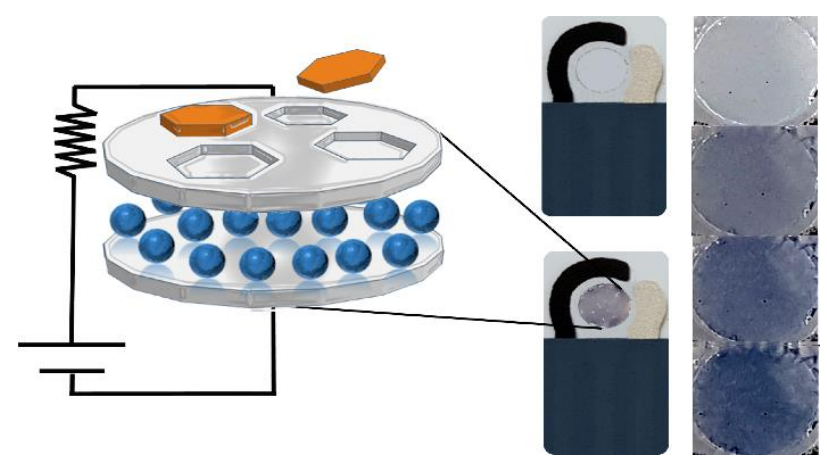

